\title{
Contenido ambientalizado y su relación con la educación química: el caso de un posgrado en enseñanza de la química
}

\author{
Conteúdo ambientalizado e sua relação com a educação química: o caso \\ de um curso na pós-graduação no ensino de química
}

\section{Environmentalist content and its relationship with chemistry education: a postgraduate in chemistry education case}

Diana Lineth Parga Lozano (dparga@ @edagogica.edu.co) Universidad Pedagógica Nacional: UPN

Diana Catalina Carrión Pérez (dccarrionp@pedagogica.edu.co) Universidad Pedagógica Nacional: UPN

Ingrid X. Arias (ingridxarias@gmail.com) Universidad Pedagógica Nacional: UPN

Resumen: La ambientalización curricular y del contenido (AC) es uno de los retos de la educación en general y de la educación química. Esta se fundamenta en implementar principios de educaciones como la ambiental (EA), la del desarrollo sostenible (EDS), la de EA y sustentabilidad en relación con la educación química (EQ). Se presentan avances de una investigación que caracterizó el contenido ambientalizado en un posgrado en enseñanza de la química, determinando sus niveles de ambientalización. Se usó metodología cualitativa e interpretativa, con análisis del contenido de los syllabus del programa entre 2010-2021. Se diseñó un instrumento para caracterizar principios de AC en la justificación, objetivos, competencias, temáticas, estrategias y referencias; validando los datos por saturación y juicio de expertos. Los resultados muestran niveles de ambientalización incipiente e intermedia en 8 de los 168 documentos analizados, es decir, hay ausencia de la $\mathrm{AC}$ en la mayoría de los documentos, quienes la abordan, lo hacen de forma individualizada, no colaborativa, a través de temas como cuestiones sociocientíficas, desarrollo sostenible, CTSA, por ejemplo. Sigue siendo un desafío en la docencia comprender dicha ambientalización por la falta de formación del profesorado y del compromiso en las políticas institucionales y nacionales.

Palabras-clave: ambientalización del contenido; ambientalización curricular; educación química; formación posgradual.

Abstract: The green curriculum (GC) is a challenge in chemistry education and education in general. This is based on implementing principles of education such as the

Recebido em: 30/05/2021

Aceite em: 13/08/2021 
environmental (EE), sustainable development (SDE), the EE \& sustainability in relation to chemistry education (CE). Progress has been shown in a research that portrayed the green content in a postgraduate in chemistry education, determining their levels. A qualitative and interpretative methodology was used with analysis of the syllabus of a program between 2010-2021. An instrument was designed to determine the GC principles in the justification, objectives, competences, thematics, strategies and references, validating data by saturation and expert judgement. Results show growing and intermediate levels of green in 8 out of the 168 documents analyzed I.e., the ones who take this approach do so in an individualistic rather than collaborative manner through thematics such as Socioscientific issues, sustainable development, STSE. It is still a challenge in education to understand such green because of a lack of education of teachers and of commitment in institutional and national politics.

Keywords: content greening; curricular greening; chemistry education; post-graduation training.

Resumo: A ambientalização curricular e do conteúdo (AC) é um dos desafios da educação em geral e da educação química. Esta AC fundamenta-se na efetivação de princípios de educações como a ambiental (EA), educação para o desenvolvimento sustentável (EDS), da EA e sustentabilidade em relação com a educação química (EQ), por citar algumas. Presentam-se avances de uma pesquisa que caracterizou o conteúdo ambientalizado em um curso de pós-graduação em ensino de química, determinando seus graus ou níveis. Foi usada a metodologia qualitativa e interpretativa, com análises do conteúdo nas ementas do curso no período 20210-2021. Foram feitos instrumentos para caracterizar princípios de AC na justificativa, objetivos, competências, temáticas, estratégias e referencias bibliográficas; validando os dados por saturação e critério de expertos. Os resultados evidenciam níveis de ambientalização fraca e intermédia em 8 dos 168 documentos analisados, isto é, ausência da AC na maioria dos documentos, os professores que a consideram, o fazem de forma individualizada, não coletiva, através de temas como questões sociocientíficas, desenvolvimento sustentável, CTSA, por exemplo. Segue sendo desafiador para a docência, compreender a ambientalização, isto, pela falta de formação do professorado e do compromisso nas próprias políticas institucionais e nacionais.

Palavras-chave: ambientalização do conteúdo; ambientalização curricular; educação química; formação na pós-graduação.

\section{INTRODUÇÃO}

En el contexto educativo, uno de los criterios influyentes en la definición de sus políticas, está definido por la UNESCO y por los problemas globales y locales actuales del mundo. La UNESCO ha establecido educar para la ciudadanía mundial (ECM) y

Recebido em: 30/05/2021

Aceite em: 13/08/2021 
cumplir los objetivos para el Desarrollo Sostenible 2030: ODS-2030. Frente a los problemas del mundo, está declarada la crisis de la humanidad en lo social, sanitario, económico, político y moral, que, para muchos, es reflejo de la crisis ambiental en la que vivimos. De acuerdo con esto, es evidente que los programas de formación docente, entre ellos los de posgrado, deben asumir tales compromisos e incluirlos como desafíos en lo que enseñamos y en la educación que hoy se quiere plantear.

En este escenario, lo enseñado por el profesorado de ciencias, al formar a sus estudiantes, puede o no estar relacionado con los problemas del mundo y en atender políticas mundiales, nacionales e institucionales, o es posible, que se enseñe, para cumplir políticas que no dan cuenta de tales necesidades. Por ello, nos preguntamos de qué manera los contenidos establecidos por el Ministerio de Educación Nacional en Colombia (MEN) para la educación química: EQ, atiende las demandas para formar en la perspectiva de la ambientalización curricular y de alguna forma, favorecer la mitigación de la crisis ambiental, esto como parte de lo formulado en la ECM. En estos desafíos, los profesores tenemos un compromiso ético y moral, sobre todo los de química, dado los impactos positivos/negativos de estos conocimientos en los diversos contextos; por ello, es otro interrogante, saber los posgrados en enseñanza de la química atienden a estas demandas.

Siendo así, en el marco de un proyecto de investigación financiado por el Centro de Investigaciones CIUP de la Universidad Pedagógica Nacional en Bogotá: proyecto CIUP DQU-548-21, se busca comprender de qué forma los contenidos curriculares de la EQ en Colombia y de un programa de posgrado, analizado como un caso, atienden las demandas actuales exigidas a la educación, siendo objeto de investigación el contenido de enseñanza del currículo de ciencias (química) del MEN y el contenido de enseñanza del currículo de una maestría en enseñanza de la química. Este análisis es pertinente porque los maestrantes que cursan el posgrado trabajan con los contenidos definidos por el MEN y llegan a la maestría con la intensión de mejorar su acción docente y con ello, la formación de sus estudiantes, por lo que también, es necesario determinar de qué forma, el contenido curricular aporta a una formación para atender los desafíos actuales para la EQ. Se parte del supuesto que el profesorado de química y las políticas nacionales aún no consideran tales perspectivas de demanda.

Recebido em: 30/05/2021

Aceite em: 13/08/2021 
Este trabajo se justifica también por la temática en sí de la ambientalización curricular y la ambientalización del contenido, AC, por los desafíos que esto demanda y porque de acuerdo con los antecedentes encontrados por Parga-Lozano (2019) son pocas las investigaciones que abordan la $\mathrm{AC}$ en la formación de profesores de química, en el pregrado y, sobre todo, en los posgrados, en el nivel de maestría.

Para el presente artículo, se enfatiza en los resultados encontrados en el posgrado, para lo cual se aborda como referente conceptual de la investigación, la línea sobre ambientalización curricular, para analizar la pertinencia del contenido enseñando: ¿será para formar en la perspectiva del presente / futuro?

Como perspectiva metodológica se hizo un análisis cualitativo e interpretativo de los documentos del programa de maestría, desde el análisis del contenido, identificando unidades de análisis que dieran cuenta de un conjunto de principios que permitieron inferir la AC.

\section{CARACTERÍSTICAS DE LA UNIVERSIDAD Y SUS DESAFIÓS}

En la literatura publicada en los últimos años si bien existe la expresión "ambientalización de la universidad", hoy es mas usada la expresión "sustentabilidad en la educación superior": SES, estos cambios obedecen, entre otras, a la política internacional sobre educación para el desarrollo sostenible (EDS) que se da entre la década de los años 90 y hasta el año 2014, posterior a esta se plantea la educación ambiental \& sustentabilidad. En este sentido, Filho (2015) considera que la SES es un campo con rápido crecimiento en la investigación y en las acciones universitarias, a pesar de ello, hay mucho por recorrer porque se viene implementando entre y en las disciplinas generalmente (Parga-Lozano, 2019).

Buscando aportar en la perspectiva de la SES, investigadores y profesores adoptan una posición estratégica para ayudar a la sociedad a aceptar soluciones sociales y técnicas para los desafíos relacionados con los problemas ambientales, asunto que requiere de soluciones integradas.

Según Filho (2015) en las instituciones de educación superior predominan tres enfoques al abordar la EDS en la educación superior: un enfoque individual en el que Recebido em: 30/05/2021

Aceite em: 13/08/2021 
los asuntos son abordados por miembros individuales de la universidad, no habiendo un colectivo para ello; el sectorial, que aborda un colectivo o una facultad, por ejemplo, comprometida con la sustentabilidad, pero sin diálogos con otros sectores de la propia institución; y el institucional, que evidencia el compromiso de toda la universidad para la EDS por estar orientado en su política universitaria.

Estos enfoques, según Parga-Lozano (2019), muestran los atrasos en las universidades frente al tema, por la falta de profesores formados para ello y la falta de interés en la temática por lo que no se adopta en la docencia ni la investigación, la no integración en los órganos de decisión universitaria que formulen objetivos estratégicos y planes de acción que den sentido a lo que se requiere y a lo que se debe hacer; es decir, es una falta de política ambiental o en sustentabilidad ambiental en la universidad, la falta de voluntad y acción para cambiar la estructura de la universidad que curricularmente funciona en torno a disciplinas, programas y facultades en las que cada uno defiende su territorio aislado de los demás. Cuando existe dicha política, no transciende a las demás esferas y se queda como una táctica y no como principios, como diría Macdonald (2015) para implementar en la docencia, la investigación y extensión universitaria.

Aspectos como los enunciados dejan ver la crisis en la universidad pública, que concordando con $\bigcirc$ Santos $€(2010)$ es un crisis hegemónica, producto de las contradicciones en las funciones universitarias: producción de cultura, de pensamiento crítico, de conocimiento científico humanístico y producción de conocimiento útil para mano de obra cualificada; es una crisis de legitimidad, porque entra en contradicción con las exigencias sociales y políticas contra la jerarquización de saberes especializados; y una crisis institucional, producto de la reivindicación de su autonomía contra las exigencias externas que les demanda eficacia, eficiencia, productividad empresarial o responsabilidad social. En términos de Chauí (2003) es una universidad operacional centrada en la transmisión, que adiestra para tener graduados para el mercado laboral e investigadores entrenados para investigar; la docencia no se enfoca en la formación, ni en la democratización, se privatiza el conocimiento habiendo exclusión social; es una universidad centrada en la gestión que orienta las demás funciones universitarias; priman los índices de productividad, sus cambios dependen de las

Recebido em: 30/05/2021

Aceite em: 13/08/2021 
demandas exigidas por el Estado u otros agentes externos, por lo que si hay política ambiental, por ejemplo, termina siendo más una táctica (Parga-Lozano, 2019).

Según lo anterior, la universidad se ha caracterizado por los siguientes aspectos:

a) La producción de conocimiento, lo que le debería exigir revisar su papel en la sociedad; producción que determina la distribución de poder económico; pero, como plantea Goergen (1998) la explosión del saber y de las capacidades humanas han hecho que se domine a la naturaleza y haya traído riesgos y problemas como los ambientales.

b) Priorizar un modelo educativo mecanicista, reduccionista, individualista y segmentado, contrario con los principios sistémicos, de complejidad y de interdisciplinariedad (MORA-PENAGOS, 2012; COMPLEXUS, 2013; PARGALOZANO, 2019) necesarios para la ambientalización y sustentabilidad ambiental.

c) Ha agudizado la hiper especialización disciplinar y la profesionalización pragmática, lo que lleva a que se asuman los problemas sociales y sus soluciones desde lo disciplinar porque el contenido curricular se define por la disciplina y no por los problemas reales y contextuales del mundo, lo que, a su vez, se ve reflejado en la organización académica de forma separada dadas las propiedades del saber específico.

d) Formación de profesionales e investigadores más individualizada que ayudan a mantener la posición hegemónica como diría Guimãres (2004), es decir, faltando la formación propia de las visiones críticas, transformadoras y emancipadoras, por ejemplo, que aportan la Educación Ambiental.

Superar estas críticas demanda de un modelo de conocimiento pluri universitario que según Santos (2010) debe ser contextual al ser aplicado fuera de la universidad interactuando productores - utilizadores; debe ser transdisciplinar por la propia aplicación, al estar en confrontación y diálogo con otros conocimientos y otros saberes, mejorando la relación ciencia naturaleza, ciencia sociedad y ciencia tecnología; no ser mercantil, sino cooperativo y solidario al establecer colaboración entre investigadores, organizaciones no gubernamentales, movimientos y grupos vulnerables, comunidades populares, ciudadanos críticos y activos; sería un conocimiento interactivo, no unilateral como lo es el modelo universitario actual (PARGA-LOZANO, 2019). Así, se podría Recebido em: 30/05/2021

Aceite em: 13/08/2021 
establecer y promover una transformación epistemológica al fomentar un diálogo de saberes, porque dialogarían las ciencias con saberes que esta misma desconoce: comunitario, popular, ancestral, campesino, tradicional, cotidiano, urbano, indígena, ..., se demanda de una justifica cognitiva que evite el epistemicidio.

No hay justicia social sin justicia cognitiva, al democratizar las prácticas sociales, ellas nunca se democratizan lo suficiente si el conocimiento que las orienta no fuera democratizado. La represión antidemocrática incluye siempre la descalificación del conocimiento y de los saberes de aquellos que son reprimidos (SANTOS, 2008, p. 111).

La universidad debe promover la sustentabilidad ambiental con ejemplos de su propia vivencia, siendo coherente con lo escrito en su política y planeación estratégica; esto es, incluir la ambientalización curricular (macro, meso y microcurricular) en todas sus funciones, enfatizando en el compromiso social, siendo pertinente, evaluando la evolución de su ambientalización, formando a su propia comunidad (estudiantes, profesores, investigadores, administrativos), siendo parte de redes, participando en la construcción de criterios de su inclusión y transformación, haciendo las reformas necesarias, lo que llevaría a una ambientalización como principio y no como táctica (MACDONALD, 2015) para que se quede solo en los documentos.

Lo anterior deja ver que a la universidad y a sus programas de formación se le cuestiona por su manera de operar y por sus funciones, a propósito de los problemas ambientales y sociales en los que estamos inmersos. Por ello preguntamos: ¿cuál es su papel en el contexto de la crisis sabiendo que la crisis del conocimiento es una crisis ambiental? ¿Cómo la universidad en general enfrenta el desafío de la ambientalización: lo hace desde la transformación epistemológica, pedagógica, didáctica, estructural y política? Si bien estas cuestiones no se resolverán en este escrito, si dejan claro que la meta de la universidad es lograr cambiar el paradigma dominante y proponer un modelo contra hegemónico. Como resultado de esto, Quintana y Mateos (2014) afirman que las universidades deben reestructurar sus funciones sustantivas, como diría Santos (2018) las universidades públicas deberían descolonizarse, despatriarcarse y desmercantilizarse, es decir, deben ser un escenario en el cual hay diversidad de conocimientos, es un espacio polifónico que trabaja por la emancipación social y no por el capitalismo, patriarcado y colonialismo académico ni político - social. Aspectos estos 
que están en la idea de la sustentabilidad y educación para la sustentabilidad (PEREIRA; AMARAL, 2020) que busca superar los reduccionismos de los modelos dominantes y crear una nueva conciencia en las personas a partir de aspectos ambientales, crisis económicas y desigualdad social (LEMKE; BARROSO, 2021).

Parga-Lozano (2019) considera que, si la universidad sigue fragmentada de la realidad social y en su interior, le será difícil su ambientalización, toda vez que es un campo que es extremo a los obstáculos de los conocimientos compartimentalizados por las disciplinas. Por ello, el gran desafío es la universidad ambientalizada en su totalidad y particularmente, en la enseñanza, al considerar en los objetivos, estrategias, competencias, temas de enseñanza, lo que significa desarticular el paradigma de cada disciplina para integrar principios de lo ambiental como parte esencial de la docencia, lo que autores como Zhou (2015) y Parga-Lozano (2019) llaman conocimiento didáctico del contenido ambientalizado. Según como esto sea hecho, se tendrán diversas formas y niveles de ambientalización: del contenido, del currículo, de la investigación, de la docencia, de la extensión, pues como dice Leff (2000), lo que se debe ambientalizar es el conocimiento mismo.

\section{AMBIENTALIZACIÓN DEL CONTENIDO Y DE LA EDUCACIÓN QUÍMICA}

En lo abordado en el numeral anterior se destacaron desafíos frente a la ambientalización en general y a la ambientalización del contenido (AC) y que son válidos para la formación posgradual y para comprender lo que sería dicha ambientalización. Parga-Lozano (2019) considera que algunos de estos desafíos tienen que ver con:

a) Abordar la AC como principio de vida académica y social (de sus comunidades), pues es necesario tener competencias de acción para la vida y su existencia en la sociedad.

b) Formar profesores para atender esta demanda.

c) Tener interés en su abordaje ya que las universidades, sus programas y profesores, tenemos un compromiso moral.

Recebido em: 30/05/2021

Aceite em: 13/08/2021 
d) Traducir la ambientalización del contenido en valores como equidad, justifica social, cognitiva y ambiental que parte del respeto en / de todo y por todos.

e) Formar / enseñar a la ciudadanía desde una nueva ética: de la integridad para conservar y cuidar la diversidad biológica y cultural; del bienestar y gobernanza para convivir en armonía; una ética con conciencia, emancipación y transformación.

f) Proponer y desarrollar una enseñanza para el mundo de la vida, lo que requiere de un aprendizaje situado y contextualizado, que supere visiones y acciones fragmentadas, disciplinares y no pertinentes para los tiempos de hoy. Esto significa hacer cambios rotundos en los planes de enseñanza, en los contenidos, en las posturas epistemológicas centradas solo en la ciencia y cuestionar los modelos hegemónicos de la sociedad, de la ciencia, de la universidad y de la enseñanza - aprendizaje.

Estos desafíos permiten comprender la ambientalización del contenido y, por lo tanto, de la enseñanza así:

Significa favorecer y mejorar las relaciones ciencia naturaleza, ciencia sociedad y ciencia tecnología; desarrollar valores de justicia (cognitiva, social, ambiental), solidaridad y equidad; conocimientos, habilidades, actitudes y valores éticos, estéticos y morales desde lo ambiental y por lo ambiental; promover la sustentabilidad desde acciones epistemológicas, metodológicas y políticas que nos permitan actuar en interacción individual colectividad (PARGA-LOZANO, 2019, p. 99).

Son diversas las conceptualizaciones acerca de la ambientalización, sobre todo la curricular, antes que la misma AC. Estas tienen que ver con las relaciones con la educación ambiental (EA), con la llamada sustentabilidad ambiental (SA), con las materias de enseñanza y con un conjunto de principios, tal como se presentan en el Cuadro 1, que resultan de las diversas revisiones de la literatura; sin embargo.

Cuadro 1 - Conceptualizaciones sobre ambientalización curricular.

\begin{tabular}{|c|c|c|c|}
\hline Relacionada con la EA & $\begin{array}{c}\text { Relacionada } \\
\text { con la SA }\end{array}$ & $\begin{array}{c}\text { Relacionada con materias } \\
\text { de enseñanza }\end{array}$ & $\begin{array}{l}\text { Relacionada con } \\
\text { principios }\end{array}$ \\
\hline $\begin{array}{l}\text { Para su integración, } \\
\text { transversalización. } \\
\text { El direccionamiento } \\
\text { ambiental de la } \\
\text { educación, su ontología. }\end{array}$ & $\begin{array}{c}\text { Transmitir sus } \\
\text { valores. }\end{array}$ & $\begin{array}{c}\text { Nuevas materias con } \\
\text { contenidos ambientales y } \\
\text { de sustentabilidad. } \\
\text { Criterios ambientales y } \\
\text { sustentables. } \\
\text { Incorporar temas en las }\end{array}$ & $\begin{array}{c}\text { Del DS, el } \\
\text { constructivismo } \\
\text { didáctico, y la } \\
\text { visión sistémica y } \\
\text { compleja asociada a } \\
\text { la naturaleza de los } \\
\text { problemas } \\
\end{array}$ \\
\hline
\end{tabular}

Recebido em: 30/05/2021

Aceite em: 13/08/2021 


\begin{tabular}{|c|c|c|c|}
\hline Relacionada con la EA & $\begin{array}{c}\text { Relacionada } \\
\text { con la SA }\end{array}$ & $\begin{array}{c}\text { Relacionada con materias } \\
\text { de enseñanza }\end{array}$ & $\begin{array}{l}\text { Relacionada con } \\
\text { principios }\end{array}$ \\
\hline \begin{tabular}{|c|} 
Su inclusión para el DS. \\
Son sus principios. \\
Su pertinencia contextual. \\
Interpretar la dimensión \\
histórica y procesual de la \\
producción ambiental. \\
Apertura de esferas \\
sociales para lo \\
ambiental; abordar las \\
dimensiones de lo \\
ambiental. \\
Abordar y comprender \\
problemas ambientales en \\
su complejidad. \\
Inclusión de las relaciones \\
ser humano, sociedad y \\
naturaleza
\end{tabular} & \begin{tabular}{|c|} 
Abordar las \\
dimensiones \\
socio \\
económicas y \\
políticas. \\
Es su abordaje \\
de forma \\
táctica o como \\
principios. \\
Incluirla \\
desde una \\
visión \\
compleja.
\end{tabular} & $\begin{array}{c}\text { disciplinas. } \\
\text { Incluir aspectos sociales al } \\
\text { currículo desde o CTS. } \\
\text { Incluir componentes del } \\
\text { ambiente. } \\
\text { Incluir la dimensión } \\
\text { ambiental o de cuestiones } \\
\text { ambientales. } \\
\text { Tiene relación con la } \\
\text { química ambiental, química } \\
\text { verde, educación para el } \\
\text { desarrollo sostenible (DS), } \\
\text { es su enseñanza; es lo } \\
\text { verde en el currículo. } \\
\text { Es analizar el contexto } \\
\text { socio ambiental. }\end{array}$ & $\begin{array}{l}\text { ambientales. } \\
\text { Ecológicos, } \\
\text { económicos y } \\
\text { sociales. } \\
\text { De la } \\
\text { sustentabilidad } \\
\text { ambiental. } \\
\text { De la dimensión } \\
\text { cultural, política, } \\
\text { técnica de la } \\
\text { educación y del } \\
\text { currículo. } \\
\text { Incluir aspectos } \\
\text { sociales, culturales } \\
\text { y ecológicos, desde } \\
\text { lo interdisciplinar y } \\
\text { multicultural. }\end{array}$ \\
\hline
\end{tabular}

Fuente. Parga-Lozano, 2019, p. 99.

Nota. DS: desarrollo sostenible; CTS: Ciencia, tecnología y sociedad; EA: educación ambiental

Para el presente análisis se asume la definición de ambientalización del contenido de Parga-Lozano (2019):

La ambientalización del contenido refiere niveles de emergencia producidos al interactuar principios del conocimiento didáctico del contenido ambientalizada, o CDC-A en lo sistémico (al tener como base el contexto en interacción con el pensamiento complejo, constructivista, hermenéutico y cibernético); crítico, holístico, flexible, con los saberes en sus diversas formas: político, económico, social, epistemológico, histórico, espiritual, ético / estético y cultural: cotidiano, científico tecnológico, ancestral, comunitario, popular; para lo cual es originada una emergencia fenomenológica de los saberes y la educación que interactúa en el salón de clase con / en y para la vida, a través del aprendizaje auto orientado, situado, colaborativo y basado en problemas y situaciones complejas de la realidad social (PARGA-LOZANO, 2019, p.109).

Además, en el contexto de la educación química, es necesario ver que esta también se ambientaliza, según Parga-Lozano y Carvalho (2019) y Parga-Lozano (2019) esto se logra desde formas como la educación química verde (EQV) y enseñanza en QV, educación química verde y química sustentable (EQV-QS), estrategias de

Recebido em: 30/05/2021

Aceite em: 13/08/2021 
sustentabilidad desde los enfoques CTS y CTSA, abordaje de controversias del DS y cuestiones sociocientíficas (CSC) y socialmente vivas (CSV), la educación química (EQ) como parte de la educación para el DS (EDS), educación química ambiental (EQA), educación en ciencia y tecnología (EC\&T), educación sobre DS, educación en ciencias de la sustentabilidad, educación para la sustentabilidad ambiental, enseñanza y aprendizaje del contenido científico con adición de temas sobre sustentabilidad, educación química para convivir en armonía, educación ambiental y sustentabilidad. En estas, según los autores citados, logran contextualizar el contenido y/o abordar diferentes niveles y formas de ambientalización.

\section{METODOLOGÍA}

Teniendo en cuenta que uno de los desafíos de la educación en ciencias naturales hoy, es la falta de ambientalización curricular y en especial, del contenido de enseñanza, se hace un estudio de caso, en un posgrado para caracterizar la AC. Este programa se escoge por ser el único de maestría en Colombia en enseñanza de la química, tiene una trayectoria de más de 30 años de funcionamiento y hoy, cuenta con más de la mitad de sus profesores con formación doctoral. De otro lado, en este nivel, existen vacíos en la investigación posgradual frente a la $\mathrm{AC}$ porque en el ámbito universitario, esta se ha centrado en los pregrados.

Para caracterizar la AC se hizo un análisis documental de 168 syllabus del período 2010-2021-1, de cada una de las materias que configuran el posgrado y se contrastaron con la política de la universidad. La información fue sometida al análisis de contenido, identificando unidades de análisis (UA): frases cortas, en relación con las unidades de contexto (párrafos) en los siguientes componentes de los programas: justificación, objetivos, competencias, temáticas, estrategias, referencias bibliográficas y evaluación. Las UA se identificaron como principios a partir de lo descrito como ambientalización curricular en el Cuadro 1 y las formas de aprendizaje citadas en el referencial. El estudio se dividió en tres fases: en la primera, se solicitaron los syllabus al posgrado y se hizo la codificación de archivos; en la segunda fase, se hizo la lectura a profundidad 
identificando en sus componentes, frases alusivas a las concepciones de ambientalización; y en la fase tres, se hizo el análisis de las tendencias encontradas.

Dado que las formas de ambientalizar, según Parga-Lozano (2019), demandan otras formas de aprendizajes, también se tuvo en cuenta esto en las UA y de contexto para identificar aspectos relacionados con preguntas críticas, pensamiento sistémico y complejo; aprendizaje aplicado, activo, participativo y colaborativo; por descubrimiento autónomo, enfocado en problemas (reales, simulados y situados), integrado, interdisciplinar y transdisciplinar, social con diversos interlocutores, avanzando hacia el diálogo de saberes, la emancipación y transformación social.

\section{RESULTADOS Y ANÁLISIS}

Revisada la política institucional de la universidad, esta promulga que la formación de profesores debe atender desafíos actuales, el compromiso con la sustentabilidad de la vida y del planeta, atender los problemas ambientales; formar con conciencia planetaria, incluir principios de sustentabilidad ambiental en los currículos, abordándolos a partir de lo interdisciplinar, el diálogo de saberes, la ética del "buen vivir", la economía solidaria y la formación de ciudadanos conscientes del compromiso con la construcción de futuro. En este contexto, el departamento que ofrece el posgrado tiene, entre otras, como misión, liderar procesos que involucren al "hombre", la cultura, la sociedad, articulados con la realidad social, cultural, económica, política y ambiental. Es decir, inicialmente, la universidad y el posgrado en enseñanza de la química, tienen principios e intenciones acordes con algunos de los criterios encontrados en las concepciones relacionadas con la ambientalización curricular.

En cuanto al programa de posgrado en enseñanza de la química, este es presencial, siendo su principal modalidad la investigación y no tanto la profundización; solo en los últimos 4 años se ha ofertado esta segunda modalidad, manteniéndose en todo caso, la de investigación. Cuenta con 50 créditos que se deben cumplir en 2 años. En la organización de los syllabus se identificó que estos atienden 3 grandes componentes. El componente asociado con la formación en didáctica de la química, con 6 seminarios o materias: conceptos químicos e implicaciones didáctica 1,2 y 3; historia y 
epistemología de la química, teorías del aprendizaje y enseñanza de las ciencias y, ciencias ambientales y educación química. El componente de investigación que corresponde al $40 \%$ de los créditos y el componente electivo en el que se desarrolla un conjunto de actividades llamadas actividades complementarias 1 y 2 , y algunos seminarios electivos.

Al analizar cada uno de los syllabus se encontró que en sus componentes de justificación, objetivos, competencias, temáticas, estrategias, evaluación y referencias bibliográficas cada uno tiene un abordaje propio de la disciplina. Tal como se evidencia en la siguiente cita para el caso de la justificación de uno de los seminarios:

Dada la necesaria formación disciplinar e investigativa a nivel de maestría, se pretende leer con los participantes la semántica de algunos conceptos químicos, las principales dificultades de enseñanza y aprendizaje; revisar (derivadas de la naturaleza propia de la química como ciencia, las estrategias de enseñanza o incluso las fuentes mismas de información) y profundizar en algunos de los conceptos básicos de la química (CQID-1_2021-1).

Como se planteó en el referente, el abordaje planeado esta en la idea de la hiper especialización disciplinar y profesional pragmática, porque se busca dar elementos conceptuales que ayuden al magister a resolver problemas de investigación del campo de la didáctica de la química, de su profesión docente, esto, se evidencia principalmente en las temáticas, justificación y competencias definidas en cada syllabus. El contenido disciplinar está determinado por las disciplinas, como por ejemplo, la psicología para el caso de teorías del aprendizaje; la química y su didáctica e historia y epistemología para conceptos químicos e implicaciones didácticas; metodología de investigación para seminarios de investigación. Criterios que hacen que a su vez haya una organización académica separada: separada por las propiedades del saber específico, si bien la química, su didáctica, su historia y epistemología son articuladoras metadisciplinares antes que interdisciplinares o transdisciplinares, pues no hay desde dicha organización un problema o metodología común para resolver una problemática.

En los aspectos de la justificación, objetivos, competencias, temas, estrategias, referencias y evaluación, definidos en los syllabus, no fueron evidentes, de forma explicita ni implícita, criterios de ambientalización del contenido: es decir, relacionados con la EA, la SA, ni principios del DS ni formas de abordar la educación química desde la EQV, EQS, EQA, ni a partir de la adición de temas sobre sustentabilidad ambiental. Recebido em: 30/05/2021

Aceite em: 13/08/2021 
El contenido está disciplinarizado. Si bien en algunos de ellos se enuncia que, por ejemplo, se abordarán los fenómenos químicos desde leyes, principios o teorías y proyectar su enseñanza desde una visión contextual e innovadora, no se precisa que sería "lo contextual", pues como se planteó, para ambientalizar el contenido, se demanda de una enseñanza - aprendizaje situado y contextualizado, si bien hay una contextualización, esta es disciplinar.

Se encontraron en total ocho seminarios con algún nivel de ambientalización: seis electivos y dos obligatorios. Dentro de los electivos, hubo tres versiones de un seminario sobre cuestiones sociocientíficas: SE-CSC y tres llamados "seminario electivo: SE1 y SE2". Dentro de los obligatorios, el seminario "Ciencias ambientales y educación química: CA-EQ" y el de "Conceptos químicos e implicaciones didácticas 3: CQID-3".

El seminario CQID-3 en el período 2014-2018 propuso como uno de sus objetivos favorecer la formación profesional del docente de química, a partir de la reflexión pedagógica y didáctica en torno a la incorporación de las relaciones Bioquímicas, Tecnología, Sociedad y Ambiente en el ámbito educativo, pero esto no se evidencia en los temas ni estrategias a desarrollar, si bien el objetivo estaría en la perspectiva de las relaciones CTSA, como una forma de ambientalizar el contenido, según Parga-Lozano (2019), sería una ambientalización puntual.

En síntesis, los criterios de una ambientalización puntual (temática) se resume en el cuadro 2.

Cuadro 2 - Criterios de ambientalización puntual en algunos componentes de los syllabus.

\begin{tabular}{|c|c|c|}
\hline Criterios & Características & Nivel de AC \\
\hline $\begin{array}{c}\text { Principios del } \\
\text { currículo }\end{array}$ & $\begin{array}{c}\text { No considera los del DS (social, científico, } \\
\text { ecológico) }\end{array}$ & N2: inicial \\
\hline Objetivos & Enfocados en cambiar comportamientos & N2: inicial \\
\hline Competencias & Desarrollar el pensamiento científico & N2: inicial \\
\hline $\begin{array}{c}\text { Disciplinas aisladas } \\
\text { Epistemología }\end{array}$ & $\begin{array}{c}\text { Las propias de los componentes de formación } \\
\text { sin interacción }\end{array}$ & N1: ausencia \\
\hline Temas & De las ciencias & N1: ausencia \\
\hline
\end{tabular}

Recebido em: 30/05/2021

Aceite em: 13/08/2021 


\begin{tabular}{|c|c|c|}
\hline $\begin{array}{c}\text { Relaciones } \\
\text { disciplinares }\end{array}$ & Multidisciplinares en algunos casos & N2: inicial \\
\hline $\begin{array}{c}\text { Didáctica y sus } \\
\text { progresiones }\end{array}$ & De las ciencias, es aislada & N1: ausencia \\
\hline Papel del profesorado & Centra la enseñanza en lo disciplinar & N1: ausencia \\
\hline Papel del alumnado & Resuelve preguntas de los temas disciplinares & N1: ausencia \\
\hline
\end{tabular}

Fuente. Elaborada a partir de Parga-Lozano (2019).

El seminario de CA-EQ en la mayoría de los componentes analizados, define desde 2017, algunos criterios de ambientalización, antes de este período no estaban. Por ejemplo, en este se establece:

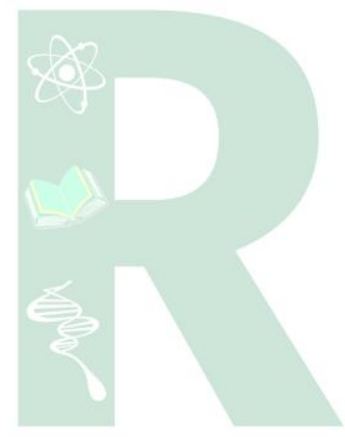

Revisar las relaciones entre el ser humano, consigo mismo y con la naturaleza, desde un análisis interdisciplinario y una perspectiva holística (Justificación: CA-EQ-2020). Profundizar en los ciclos biogeoquímicos, para comprender las formas de conservación y el desarrollo sostenible (Objetivos: CA-EQ-2020). El reconocimiento de debates actuales, la interpretación de modelos químico y la identificación del papel de la pregunta, materiales didácticos y estrategias metodológicas vinculadas con la resolución de problemas socio ambientales, desarrollo científico y tecnológico en cuestiones socialmente vivas. (Competencias: CA-EQ-2020) Resaltado nuestro.

Del 2010 al 2016 los contenidos fueron sobre el estudio de la organización de la materia, en los niveles macro, micro y super macromundo, dinámica terrestre, estructura interna terrestre, dinámica de los materiales terrestres, estructura de los materiales terrestres, corteza terrestre, geociclos y ciclo genético de las rocas, sistemas biológicos suelos, biosfera, ecosfera, sistema atmosférico, sistema hidrológico, equilibrio ecológico y parques de Colombia. A partir de 2017 se trabajó sobre modelos en ciencia y enseñanza y aprendizaje de las ciencias, los aportes desde la termodinámica alejada del equilibrio, comprensión de los seres vivos y enseñanza bioquímica de la complejidad desde un enfoque contextualizado. Finalmente, en el segundo semestre de 2020 con el cambio del docente, se abordan aspectos de los niveles de organización de la materia según tamaño y función (conceptos básicos de las ciencias y del ambiente); dinámica terrestre: ecosistemas, sus problemas e impactos del hombre en los ecosistemas, perspectivas desde la química, suelo, fertilidad, ciclo de energía; sistema atmosfera e hidrósfera: la atmósfera, el clima, la hidrósfera, calidad y fragilidad del agua y el aire, y

Recebido em: 30/05/2021

Aceite em: 13/08/2021 
los cuestionamientos acerca de la problemática social y científica asociada al cambio climático y al calentamiento global; el sistema global ambiental y la educación para el desarrollo sostenible (visión global del mundo, interacciones ciencia-tecnología, problemas socio científicos relacionados con la biotecnología y el ambiente.

Estos temas, en la mayoría de los casos, están en la perspectiva de las ciencias ambientales y su relación con las ciencias naturales, por lo que se favorece una visión ecologizada de situaciones geológicas y disciplinares de la química y la geociencia. Es decir, se abordan contenidos sobre, no se enseña desde principios de la EDS, o de la EA, o la educación para la sustentabilidad ambiental, o la epistemología ambiental, por ejemplo.

Respecto a los seminarios electivos, el de cuestiones sociocientíficas (SE-CSC), se ofertó durante tres semestres (2011-1, 2013-2 y 2014-1); buscó profundizar en una alternativa didáctica para trabajar la naturaleza de la ciencia y la tecnología, la toma de decisiones, la argumentación y el razonamiento ético y moral en los estudiantes; analizar el uso de cuestiones sociocientíficas (CSC) en las clases de ciencias; analizar y evaluar el desarrollo de proyectos de aula sobre CSC; en el 2014, se hizo énfasis en el análisis del uso de cuestiones sociocientíficas y ambientales en las clases de ciencias desde un proyecto de aula o un proyecto de investigación. Para cumplir con estos objetivos, se propusieron temas sobre CTSA, CSC en la enseñanza y formación del profesor investigador, historia del movimiento CTS y CTSA, análisis crítico de currículos CTSA, tendencias de los enfoques, emergencia de las CSC, características y proyectos sobre CSC y ambientales, evaluación de estos diseños curriculares.

En 2015, con el SE2 se propuso el planteamiento de situaciones problema en un contexto controvertido, para favorecer el desarrollo del conocimiento profesional docente a partir del diseño de secuencias didácticas en torno a controversias sociocientíficas o socialmente vivas. Sin embargo, Los temas estuvieron más en la perspectiva disciplinar química: transformaciones, estructura de la materia, teoría de enlace de valencia y del orbital molecular, geometría molecular, reacción química, cantidad de sustancia (mol), propiedades de las sustancias, implicaciones didácticas del concepto de reacción química, enlace, cantidad de sustancia desde la perspectiva CTSA; conceptualización de alimento, nutriente, sustancias, aditivo, metabolismo, 
microorganismos usados en la industria de alimentos, procesos tecnológicos: deshidratación, fermentación, emulsión, osmosis, osmosis inversa, mezclas, soluciones, cambios de fase, control de calidad fisicoquímica, microbiológica y organoléptica, agua fuente de vida en peligro de muerte, los aditivos alimentarios conservan o deterioran, los agrotóxicos control biológico o social, diseño de secuencias y/o unidades didácticas.

Luego, en 2018-1 y 2019-1 se desarrollaron, dos versiones del SE1. Para el 20181 se propuso el desarrollo de competencias para comprender la complejidad de los problemas ambientales de contenido científico, técnicos, normativos, sociales, económicos políticos y culturales en la realidad educativa. En el 2019-1, se plantearon competencias para comprender la complejidad de los problemas ambientales desde lo histórico, social, económico, político y cultural. Los contenidos estuvieron en relación con las ciencias ambientales como fundamento para mejorar las relaciones ser humano ambiente, la educación ambiental desde la contaminación con cromo, educación ambiental y desarrollo humano, experiencias significativas, políticas ambientales, agenda 21, agenda 2030, educación y sostenibilidad. En 2019 se enfatizó en educación ambiental, políticas ambientales, pensamientos y construcciones, compromisos de los profesores, relaciones entre educación ambiental y enseñanza de la química.

De acuerdo con lo descrito, los seminarios electivos del programa de posgrado son los que han desarrollado aspectos de educación en ciencias y didáctica de las ciencias con implicaciones de ambientalización del contenido, aunque de forma implícita para el seminario que desarrolló el tema de cuestiones sociocientíficas y cuestiones socialmente vivas, y más explícitamente con los seminarios de 2018 y 2019. En el caso de los seminarios obligatorios, la perspectiva está desde las ciencias ambientales, pero en todos los casos, son temas para enseñar, es decir, se enseña sobre educación ambiental, EDS, abordaje del CTSA educativo, CSC, cuestiones ambientales, contenido científico con adición de temas sobre sustentabilidad, lo que es importante para comprender estas educaciones e implicaciones y su relación con la educación y enseñanza de la química. En este caso, hay una ambientalización disciplinar más que puntual.

En general, se plantea que si en algún componente del syllabus, en la justificación, objetivos, competencias, temáticas o estrategias de enseñanza, etc., hay alguna mención,

Recebido em: 30/05/2021

Aceite em: $13 / 08 / 2021$ 
o está implícito alguna forma de relación de las expresadas en el Cuadro 1, existe una ambientalización del contenido puntual (Cuadro 2), la cual es aún inicial o incipiente. En tanto que en 8 seminarios electivos se encontraron aspectos de una ambientalización disciplinar, dado que la mayoría de sus componentes alude a las relaciones y concepciones expresadas en el Cuadro 1.

\section{CONSIDERACIONES FINALES}

Si bien la universidad tiene en parte de su política, en el plan de desarrollo institucional, aspectos que dan cuenta de principios de ambientalización curricular, se esperaría que esto favoreciera los programas de formación, en especial los posgrados, alguna forma de ambientalización, que entre otras, busca superar la fragmentación disciplinar por la perspectiva interdisciplinar, pero por lo evidenciado, esto no es posible, toda vez que la organización curricular y la administración académica está pensada desde disciplinas; más, qué tanto impide esto a la universidad para reinventarse, como afirma González-Gaudiano (2009). De qué forma los principios de la política del programa y de la universidad, son asumidos en la planeación de cada espacio académico de la maestría y por lo tanto, en su docencia, pues estos elementos de política se vuelven aspectos de lo deseable junto con lo expresado en el referencial del presente escrito, al considerar otras relaciones disciplinares: interdisciplinar y transdisciplinar para ir hacia el diálogo de saberes; al considerar otras formas de aprendizaje, al contextualizar la enseñanza; al considerar el DS y el DHS, los principios de lo sistémico, la sustentabilidad misma para atender la formación posgradual para la enseñanza de la química que considera formar sobre y desde EA y EDS, en la medida que los magísteres ayuden en la transformación social y para lo cual, deben dar cuenta de otras competencias demandadas para la sustentabilidad ambiental.

De los 168 syllabus del período 2010-2021-1 analizados, en 160 de estos, el contenido del programa de maestría se desarrolla desde las relaciones disciplinares consigo misma, enfocados desde la epistemología de la ciencia y por lo tanto, desde visiones lineales; están centrados más en sus políticas internas, sin considerar las políticas de la propia universidad y las externas que demandan ambientalizar el

Recebido em: 30/05/2021

Aceite em: 13/08/2021 
contenido. En cuanto a las competencias son disciplinares, propenden por el desarrollo del pensamiento objetivo, antes que el crítico, sistémico o complejo; la formación está centrada en el individuo quien resuelve preguntas de la enseñanza de la química, esto es disciplinar, por estar fundamentada en la didáctica de la química. El contenido es temático y monodisciplinar, fragmentado y abstracto. Tales características hacen que se planee una enseñanza poco ambientalizada, o si se quiere, una ambientalización débil toda vez que aparece en la misión y visión del programa y de la universidad principios de ambientalización, pero que en la planeación de la docencia no se refleja aún.

Fue en 8 seminarios electivos en los que se encontró una ambientalización puntual y otra disciplinar. La ambientalización puntual se dio porque se propusieron en los objetivos, competencias o temáticas principalmente, aspectos que se identifican dentro de las concepciones de AC. Las competencias buscan desarrollar más el pensamiento científico para comprender los problemas de naturaleza ecológica que ambiental; los temas si bien traen aspectos sobre EA, DS, CTSA, son temas para desarrollar más que principios, mecanismo de enseñanza, esto deriva en una ambientalización incipiente en el contenido, lo que coincide con lo encontrado en el pregrado por Parga-Lozano, MoraPenagos y Cárdenas (2013; 2014); Rodríguez-Zamudio (2015); Guerra et al. (2015); es decir, el posgrado es reflejo de lo que sucede en el pregrado en cuanto a la AC.

La ambientalización disciplinar se da a través de una materia como tal para desarrollar contenidos relacionados con lo ambiental o sustentabilidad ambiental, pero son seminarios electivos; sus principios están en la mayoría de los componentes del propio syllabus; sin embargo, en ninguno de estos se proponen competencias en la perspectiva de las demandas para el siglo XXI: pensamiento sistémico, anticipatorio, de compromiso social, estratégico y ético, las de acción, conexión e interdependencia como establecen Grecho (2008), Mora-Penagos (2012); Sjöström; Rauch y Eilks, (2015); Wals y Dillon, 2015; Novo y Murga-Menoyo (2015); Barth (2016). Se plantearon competencias que promueven en algunos casos, el pensamiento crítico, siendo el magister quien puede resolver problemas disciplinares (químicos y didácticos) con implicaciones de la EA y la EDS principalmente.

Las temáticas tienen un enfoque interdisciplinar, con abordajes de una epistemología de la ciencia y ambiental, con propósitos para cumplir políticas del Recebido em: 30/05/2021

Aceite em: 13/08/2021 
programa, por ejemplo al analizar las relaciones CTS, CTSA, formar para la comprensión de problemas ambientales, involucrando la relación cultura, sociedad con la realidad social y ambiental;; sin embargo el contenido y las estrategias de enseñanza como están descritas, es desde la disciplina (un seminario electivo: por ejemplo, el seminario de CSC), más que desde los problemas del contexto real y situado. Estas condiciones permiten deducir bajo los criterios de Parga-Lozano (2019) que la ambientalización es disciplinar, por lo que estarían en un nivel intermedio.

Los que desarrollan estas visiones son profesores individuales, que han tenido en su formación doctoral principalmente, conocimiento de estas perspectivas y comenzaron a investigar y enseñar sobre ello. Burmeister y Eilks (2014); Stratton et al. (2015); Macdonald (2015) consideran que los enfoques del DS y del desarrollo humano sustentable (DHS) son poco conocidos y por ello no se enseñan, por lo que se debe trabajar más en las universidades, esto hace que haya una deficiencia en la educación para el DS, como lo afirman Filho (2015) y Filho et al. (2017) y para una educación ambiental y sustentabilidad (EAyS) como lo afirma Wals (2012).

Si bien la universidad tiene aspectos de la política que llaman a ambientalizar el currículo, el contenido y la docencia, es responsabilidad de los docentes poderla desarrollar, pero también de la universidad, el formar a sus propios profesores para lograrlo, ejemplo de esto es lo que ha comenzado a hacer de forma individual los profesores que conocen estas perspectivas y comienzan a enseñarlas, ojalá para ambientalizar el contenido de forma más abarcadora y no solo puntual o como una materia de más.

\section{REFERENCIAS}

BARTH, M. Teaching and Learning in Sustainability Science. In: HEINRICHS, H.; MARTENS, P.; MICHELSEN, G.; WIEK, A. (Eds.). Sustainability Science an introduction (pp. 325-333). New York: Springer Dordrecht Heidelberg. 2016.

BURMEISTER, M.; EILKS. I. Issues of sustainable development and green chemistry for innovating secondary chemistry teacher education. In: CONSTANTINOU, C. 2012. PAPADOURIS, N.; HADJIGEORGIOU, A. (Ed.). European science education research association: The ESERA 2013 Conference: Strand 2, Learning science: Cognitive, affective and social aspects (pp. 438-445). Nicosia, Chipre. 2014.

Recebido em: 30/05/2021

Aceite em: $13 / 08 / 2021$ 
CHAUÍ, M. A universidade pública sob nova perspectiva. Revista Brasileira de Educação, n. 24, 5-15, set. / dez. 2003.

COMPLEXUS. Indicadores para medir la Contribución de las Instituciones de Educación Superior a la Sustentabilidad. León, Guanajuato, México: Universidad de Guanajuato. 2013.

FILHO W.L. Transformative Approaches to Sustainable Development at Universities. Working Across Disciplines. Switzerland: Springer International Publishing. DOI 10.1007/978-3-319-08837-2. 2015.

FILHO, W. L. AZEITERO, U. M.; ALVES, F.; MOLTHAN, P. (Eds.). Handbook of Theory and Practice of Sustainable Sustainability Series. Cham, Switzerland: Springer international Publisher. v.4. DOI 10.1007/978-3-319-47877-7_32. 2017.

GONZÁLEZ-GAUDIANO, E. La educación ambiental institucionalizada: actos fallidos y horizontes de posibilidad. Perfiles educativos. v.31, n.124. 58-68. 2009.

GOERGEN, P. Ciência, sociedade e universidade. Educação e Sociedade, v.19, n.63, Campinas, ago. Doi.org/10.1590/S0101-73301998000200005. 1998.

GRECHO, A. S. For Determining criteria and indicators for success in higher education programs focused on environmental sustainability. Tese (doutorado) Programa de Silvicultura. North Carolina State University, North Carolina: United States. 2008.

GUERRA, A. F. S.; FIGUEIREDO, M. L.; ORSI, R. F. M.; STEUCK, E. R.; CARLETTO, D. L.; SILVA, M. P.; LUNA, J. M. A ambientalização na Educação Superior: trajetória e perspectivas. In: GUERRA, A. F. S. (Org.). Ambientalização e sustentabilidade nas universidades: subsídios, reflexões e aprendizagens (pp. 1133). Itajaí: UNIVALI Editora. 2015.

GUIMARÃES, M. Educação ambiental crítica. In: LAYRARGES, P. P. (Coord.). Identidades da Educação ambiental no Brasil, (pp. 25-35). Brasília: Ministério do Meio Ambiente. 2004.

LEFF, E. Pensar la complejidad ambiental. In: LEFF, E. (Coord.). La complejidad ambiental (pp. 7-53). México: Siglo XXI editores, s. de c.v. 2000.

LEMKE, C.S.; BARROSO, P.F. Percepções sobre sustentabilidade dos estudantes do IFSul Campus Pelotas - Visconde da Graça (CaVG). Revista Insignare Scientia, v.4, n. 1, p. 189-205. Abril, 2021.

MACDONALD, M. Sustainability and science education: modes of pedagogic practice. In: ACHIAM, M.; CARVALHO, G. (Eds.). European science education research association: The ESERA 2015 Conference. Part 9, Strand 9, environmental, health and outdoor science education (pp. 1338-1349). Helsinki, Finland. 2015.

MORA-PENAGOS, W. M. Ambientalización curricular en la educación superior: un estudio cualitativo de las ideas del profesorado. Profesorado: revista de currículo y formación del profesorado. v.6, n.2, 77-103. 2012.

NOVO, M.; MURGA-MENOYO, Mª A. The Processes of Integrating Sustainability in Higher Education Curricula: A Theoretical-Practical Experience Regarding Key

Recebido em: 30/05/2021

Aceite em: 13/08/2021 
Competences and Their Cross-Curricular Incorporation into Degree Courses. In: FILHO, W.L. (Ed.). Transformative Approaches to Sustainable Development at Universities (pp. 119-133). Hamburg, Germany: Springer. 2015.

PARGA-LOZANO, D. L.; CARVALHO, W. L. P. A pesquisa sobre ambientalização curricular. Revista de la facultad de ciencia y tecnología -Tecné, Episteme y Didaxis: TED, (46), 39-56. 2019. DOI: https://doi.org/10.17227/ted.num46-10539 (2019).

PARGA-LOZANO, D. L.; MORA-PENAGOS, W. M.; CÁRDENAS, Y. Dimensión ambiental, una inclusión necesaria para la formación de profesores de ciencias.

Góndola, Enseñanza y aprendizaje de las ciencias, v.9, n.1, 38-46. 2014.

PARGA-LOZANO, D.L. Conhecimento didático do conteúdo ambientalizado na formação inicial do professor de química na Colômbia. Tese (Doutorado em Educação para a Ciência). Programa de Pós-Graduação em Educação para a Ciência, Faculdade de Ciências, Universidade Estadual Paulista-UNESP-, "Júlio de Mesquita Filho", Bauru/SP, 2019. Disponible en: http://hdl.handle.net/11449/190931

PEREIRA, V.A.; AMARAL, M.J. Novas exigências a educação ambiental no contexto pós-COVID-19: Desafios a redefinição do projeto pedagógico. Revista Insignare Scientia, v.3, n. 5, p. 312-326. Set/Dez. 2020.

QUINTANA, G. E.; MATEOS, J. E. G. ¿Incluir contenidos ambientales o formar con una perspectiva ambiental? RIPS: Revista de investigaciones políticas y sociológicas, v.13, n.2, 123-137. 2014.

RODRÍGUEZ-ZAMUDIO, C. Educación ambiental en la educación superior.

Consideraciones teóricas y metodológica. Bogotá: UD Editorial, Universidad Distrital Francisco José de Caldas. 2015.

SANTOS, B. S. Debates sobre la universidad en el siglo XXI. In: TAPIA, L.; MIRTENBAUM, J.; RODRÍGUEZ, G.; MAYORGA, F.; SALAZAR, C.; MARIACA, G.; PATZI P. F.; SANTOS, B. S. Pensar el Estado y la sociedad: desafíos actuales (pp. 37-99). La Paz: Muela del diablo editores, Comuna CLACSO. 2008.

SANTOS, B.S. Um discurso sobre as ciências. São Paulo: Cortez Editora. 2010.

SANTOS, B.S. Universidades públicas deben desmercantilizarse, descolonizarse y despatriarcalizarse. 24 de abril de 2018. Disponible en:

<https://www.ucr.ac.cr/noticias/2018/04/24/universidades-publicas-debendesmercantilizarse-descolonizarse-y-despatriarcalizarse.html

SJÖSTRÖM, J.; RAUCH, F.; EILKS, I. Chapter 9: Chemistry education for sustainability. In: EILK, I. S; HOFSTEIN. A. Relevant Chemistry Education: From Theory to Practice (pp. 163-183). The Netherlands: Sense publishers: Rotterdam. 2015.

STRATTON, S.K.; HAGEVIK, R.; FELDMA, A.; BLOOM, M. About this book. In: STRATTON, S.K; HAGEVIK, R.; FELDMAN, A.; BLOOM, M. (Eds). Educating Science Teachers for Sustainability (pp xiii-xvi). Switzerland: Springer International Publishing AG. 2015.

Recebido em: 30/05/2021

Aceite em: $13 / 08 / 2021$ 
WALS, A.; DILLON, J. Foreword. In: STRATTON, S.K; HAGEVIK, R.; FELDMAN, A.; BLOOM, M. (Eds.). Educating Science Teachers for Sustainability (pp. v-vii). Switzerland: Springer International Publishing AG. 2015.

WALS, A.E.J. Learning our way out of un-sustainability: the role of environmental education. In Oxford Handbook on Environmental and Conservation Psychology, Clayton, S. London: Oxford University. 2012.

ZHOU. G. Chapter 11. Environmental Pedagogical Content Knowledge: A Conceptual Framework for Teacher Knowledge and Development. In: STRATTON, S.K;

HAGEVIK, R.; FELDMAN, A.; BLOOM, M. (Eds). Educating Science Teachers for Sustainability (pp. 185-203). Switzerland: Springer International Publishing AG. 2015.

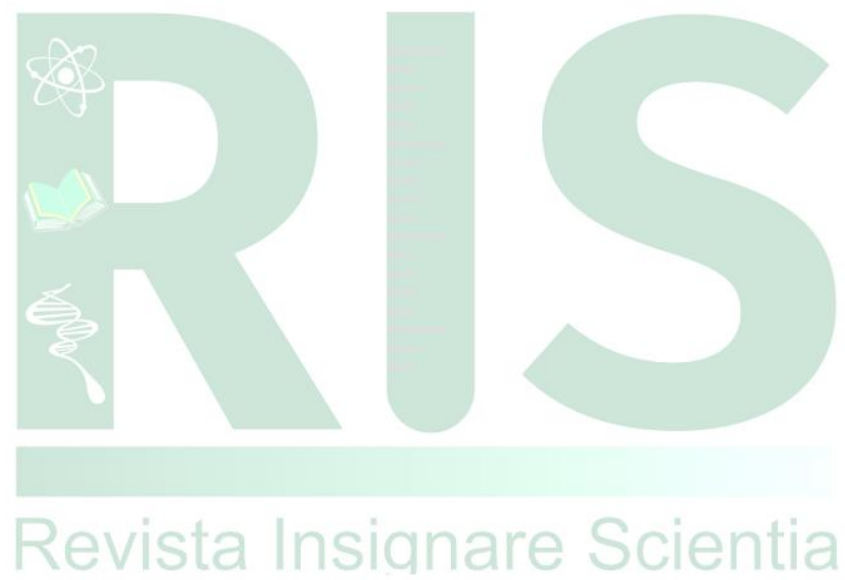

Recebido em: 30/05/2021

Aceite em: 13/08/2021 